\title{
Modeling the DBR laser used as wavelength conversion device
}

\author{
Braagaard, Carsten; Mikkelsen, Benny; Durhuus, Terji; Stubkjær, Kristian
}

Published in:

Journal of Lightwave Technology

Link to article, DOI:

10.1109/50.296182

Publication date:

1994

Document Version

Publisher's PDF, also known as Version of record

Link back to DTU Orbit

Citation (APA):

Braagaard, C., Mikkelsen, B., Durhuus, T., \& Stubkjær, K. (1994). Modeling the DBR laser used as wavelength conversion device. Journal of Lightwave Technology, 12(6), 943-951. https://doi.org/10.1109/50.296182

\section{General rights}

Copyright and moral rights for the publications made accessible in the public portal are retained by the authors and/or other copyright owners and it is a condition of accessing publications that users recognise and abide by the legal requirements associated with these rights.

- Users may download and print one copy of any publication from the public portal for the purpose of private study or research.

- You may not further distribute the material or use it for any profit-making activity or commercial gain

- You may freely distribute the URL identifying the publication in the public portal

If you believe that this document breaches copyright please contact us providing details, and we will remove access to the work immediately and investigate your claim 


\title{
Modeling the DBR Laser Used as Wavelength Conversion Device
}

\author{
Carsten Braagaard, Benny Mikkelsen, Terji Durhuus, and Kristian E. Stubkjaer, Member, IEEE
}

\begin{abstract}
In this paper, a novel and efficient way to model the dynamic field in optical DBR-type semiconductor devices is presented. The model accounts for the longitudinal carrier, photon, and refractive index distribution. Furthermore, the model handles both active and passive sections that may include gratings. Thus, simulations of components containing, e.g., gain sections, absorptive sections, phase sections, and gratings, placed arbitrarily along the longitudinal direction of the cavity, are possible.

Here, the model has been used for studying the DBR laser as a wavelength converter. Particularly, to improve the performance of the DBT converter, the influence of systezm and device parameters will be discussed. Calculations show that ultrafast wavelength conversion with rise and fall times less than 50 ps can be obtained. Also, a regenerative effect simultaneous with the wavelength conversion is expected, e.g., improvements in the extinction ratio of the signal of more than $6 \mathrm{~dB}$ is predicted for correct design and operation of the component.
\end{abstract}

\section{INTRODUCTION}

D EVICES with all-optical wavelength conversion capabilities will be essential elements in future WDM systems, e.g., as key components in photonic switching architectures [1]. Recently, penalty-free wavelength conversion of $5 \mathrm{~Gb} / \mathrm{s}$ signals has been demonstrated by the use of optical carrier modulation of a distributed Bragg reflector (DBR) laser [2]. Several other wavelength conversion mechanisms have been proposed, such as four-wave mixing, gain saturation, and cross-phase modulation in semiconductor optical amplifiers [3]-[5]. Also, the absorptive bistability in DBR lasers has been applied for the conversion of the optical wavelength [6], and $Y$-lasers have been proposed as wavelength conversion devices [7] as well. The operating speed of a converter based on semiconductor optical amplifiers or components with absorptive sections is limited by a long carrier lifetime. Therefore, the bit rate for such a converter is restricted to a few gigabits/second. On the other hand, wavelength conversion by carrier modulation in lasers is advantageous at high bit rates since the operating speed is only limited by the relaxation frequency of the laser.

In this paper, an advanced dynamic large-signal model is presented, capable of modeling the carrier dynamics and the field evolution in, e.g., optical semiconductor amplifiers, DFB structures, or DBR lasers, as well as in even more complicated and complex integrated components. The model is used for

Manuscript received July 8, 1993; revised January 21, 1994.

The authors are with the Centre for Broadband Telecommunications, Electromagnetics Institute, Technical University of Denmark, DK-2800 Lyngby, Denmark.

IEEE Log Number 9401715. studying a tunable DBR laser as a wavelength converter. The modeling reveals the influence of important device parameters and allows optimization of the DBR converter. It will be shown that efficient, ultrafast wavelength conversion with rise and fall times less than 50 ps can be obtained by correct design and operation of the converter.

This paper is organized as follows. A description of the numerical model is presented in Section II. In Section III, the mode of operation is described for the DBR laser used as a wavelength converter. Predictions of the model for DBR converters are presented in Section IV, and finally, Section V gives a brief conclusion.

\section{NUMERICAL MODEL}

In order to acquire knowledge of the carrier dynamics in optical semiconductor devices, a detailed large-signal model is developed, taking the carrier and optical field distributions into account. The model handles structures with changing device and material parameters along the longitudinal direction of the cavity. This enables assessment of components combined with, e.g., gain sections, absorptive sections, phase sections, as well as gratings.

Similar to previously developed models published in [8] and [9], this numerical model handles the nonuniform distribution of carriers and photons by splitting the device cavity into subsections, each with a constant carrier density and thereby a constant wavenumber. Then the field distributions in the longitudinal direction of the cavity for the forward and backward traveling fields are found from a transmission line description.

Besides the simulation of split electrode devices with inhomogeneous current injection, the sectioning of the device gives the possibility of investigating phenomena such as spatial hole burning and gain saturation effects. Also, multichannel crosstalk and bistability due to absorption and dispersion can be described. The model also includes the facet reflectivities, which influence the carrier and field distribution, and it handles arbitrary time-varying current modulations as well as arbitrary optical input waveforms.

A sectioning of the cavity into $n$ subsections is assumed, as shown in Fig. 1. The subsections are separated by reference planes, numbered from 0 to $n$, with reference plane number $m$ placed at the position $z_{m}$ in the longitudinal direction of the cavity. The subsection at the left and right sides of reference plane $m$ are denoted section $m_{-}$and $m_{+}$, respectively. In each subsection, it is assumed that the carrier density, photon density, and material parameters are constant. However, the carrier and photon density vary from subsection to subsection, 


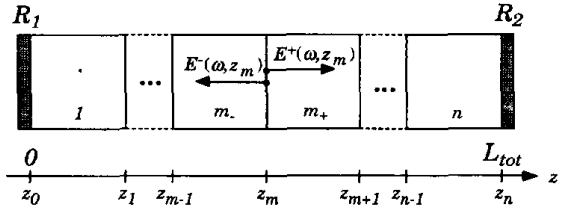

Fig. 1. Schematic diagram of an optical semiconductor device, with the cavity divided into $n$ subsections. $E^{ \pm}$denotes the electrical fields, $R_{1}$ and $R_{2}$ are facet reflectivities, and $L_{\text {tot }}$ is the total length of the cavity.

and the material parameters can change from one subsection to another.

At reference plane number $m$, the field amplitudes $F^{-}\left(t, z_{m}\right)$ and $F^{+}\left(t, z_{m}\right)$ of the left and right traveling fields, respectively, can be derived from Maxwell's equations using the slowly varying envelope approximation. However, the fields $F^{ \pm}\left(t, z_{m}\right)$ are not unique at the boundary $z_{m}$ if discrete reffections occur or if the grating period is changed. In that case, the fields must be calculated immediately outside the reference plane.

If the device contains a grating in either the full length or a part of the device, dynamic mode-coupled equations are necessary to investigate the transient behavior of the structure. As shown in the Appendix, these time-dependent coupled equations can be written as

$$
\begin{aligned}
& \frac{\partial F^{ \pm}\left(t, z_{m}\right)}{\partial z} \\
& \mp\left[g_{m_{ \pm}}-j\left(\frac{\Gamma_{m_{ \pm}}}{2} \frac{\partial g}{\partial \omega}-j \frac{\partial \beta}{\partial \omega}\right) \frac{\partial}{\partial t}\right] F^{ \pm}\left(t, z_{m}\right) \\
& =\mp\left[\kappa_{m_{ \pm}}-\frac{\partial \kappa}{\partial \omega} \frac{\partial}{\partial t}\right] e^{ \pm 2 j\left(\beta_{m_{ \pm}}-\beta_{0, m_{ \pm}}\right) z_{m}} F^{\mp}\left(t, z_{m}\right) .
\end{aligned}
$$

Here, $\beta_{m_{ \pm}}$is the real part of the wavenumber, $g_{m_{ \pm}}$is the modal gain for the field, $\Gamma_{m_{ \pm}}$is the confinement factor for the intensity of the fundamental waveguide mode, $\beta_{0, m_{ \pm}}$denotes the Bragg wavenumber, and $\kappa_{m_{ \pm}}$is the grating coupling coefficient that governs the distributed feedback in subsections $m_{-}$and $m_{+}$, respectively.

By using a large number of subsections, it is possible to introduce the following equations to describe the partial derivative of the fields with respect to time and the $z$ coordinate:

$$
\begin{aligned}
& \frac{\partial F^{ \pm}\left(t, z_{m}\right)}{\partial t} \approx \frac{F^{ \pm}\left(t, z_{m}\right)-F^{ \pm}\left(t-\tau_{m_{\mp}}, z_{m \mp 1}\right)}{\tau_{m_{\mp}}} \\
& \frac{\partial F^{ \pm}\left(t, z_{m}\right)}{\partial z} \approx \frac{F^{ \pm}\left(t, z_{m}\right)-F^{ \pm}\left(t-\tau_{m_{\mp}}, z_{m \mp 1}\right)}{L_{\mathrm{sec}}} .
\end{aligned}
$$

It should be noticed that the approximations of the derivatives each include a change of both time and place, which may affect the propagation delay effects. $L_{\mathrm{sec}}$ is the length of each subsection given by $L_{\mathrm{sec}}=L_{\mathrm{tot}} / n$, where $L_{\text {tot }}$ is the total length of the device. $\tau_{m_{ \pm}}$is the transit time of the field for subsection number $m_{+}$and $m_{-}$, respectively, which can be written as

$$
\tau_{m_{ \pm}}=\frac{L_{\mathrm{sec}}}{V_{g, m_{ \pm}}} .
$$

$V_{g, m_{ \pm}}=c / n_{g, m_{ \pm}}$is the group velocity, and the group index $n_{g, m_{ \pm}}$is given by the following Taylor expansion:

$$
n_{g, m_{ \pm}}=n_{m_{ \pm}}+\omega \frac{\partial n}{\partial \omega} .
$$

$n_{m_{+}}$is the carrier-density-dependent effective refractive index in subsection number $m_{+}$and $m_{-}$, and $\partial n / \partial \omega$ is assumed to be constant.

Inserting (2a) and (2b) into (1) and after some algebraic manipulations, it is possible to separate and find the amplitudes of the forward and backward traveling fields at the place $z_{m}$ to the time $t$ by solving the two equations with the two unknown fields $F^{+}\left(t, z_{m}\right)$ and $F^{-}\left(t, z_{m}\right)$ :

$$
\begin{aligned}
F^{+}\left(t, z_{m}\right) & =\frac{\operatorname{det}\left(\begin{array}{ll}
C_{m}^{+} & B_{m}^{-} \\
C_{m}^{-} & A_{m}^{-}
\end{array}\right)}{\operatorname{det}\left(\begin{array}{ll}
A_{m}^{+} & B_{m}^{-} \\
B_{m}^{+} & A_{m}^{-}
\end{array}\right)}, \\
F^{-}\left(t, z_{m}\right) & =\frac{\operatorname{det}\left(\begin{array}{ll}
A_{m}^{+} & C_{m}^{+} \\
B_{m}^{+} & C_{m}^{-}
\end{array}\right)}{\operatorname{det}\left(\begin{array}{ll}
A_{m}^{+} & B_{m}^{-} \\
B_{m}^{+} & A_{m}^{-}
\end{array}\right)} .
\end{aligned}
$$

In (5), det represents the determinant, and the following quantities have been introduced in order to simplify the notation:

$$
\begin{aligned}
A_{m}^{ \pm}= & \pm \frac{1}{L_{\mathrm{sec}}} \mp g_{m_{ \pm}} \pm \frac{1}{\tau_{m_{\mp}}}\left(j \frac{\partial g}{\partial \omega}+\frac{1}{V_{g, m_{ \pm}}}\right) \\
B_{m}^{ \pm}= & \left(j \kappa_{m_{ \pm}}+\frac{1}{\tau_{m_{\mp}}} \frac{\partial \kappa}{\partial \omega}\right) e^{\mp j 2\left(\beta_{m_{ \pm}}-\beta_{0, m_{ \pm}}\right)} \\
C_{m}^{ \pm}= & \pm\left(\frac{1}{L_{\text {sec }}}+\frac{1}{\tau_{m_{\mp}}}\left(j \frac{\partial g}{\partial \omega}+\frac{1}{V_{g, m_{\mp}}}\right)\right) \\
& \cdot F^{ \pm}\left(t-\tau_{m_{\mp}}, z_{m \mp 1}\right) \\
& \pm\left(\frac{1}{\tau_{m_{ \pm}}} \frac{\partial \kappa}{\partial \omega}\right) e^{ \pm j 2\left(\beta_{m_{\mp}}-\beta_{0, m_{\mp}}\right) z_{m}} \\
& \cdot F^{\mp}\left(t-\tau_{m_{ \pm}}, z_{m \pm 1}\right) .
\end{aligned}
$$

The boundary condition for the forward traveling field at reference plane number $m=0$ is given by

$$
F^{+}\left(t, z_{0}\right)=\sqrt{R_{1}} F^{-}\left(t, z_{0}\right)+\sqrt{1-R_{1}} F_{\text {in }}(t)
$$

where $F_{\text {in }}(t)$ is the injected input field and $R_{1}$ is the front facet reflection. In the case of $m=n$, the boundary condition for the backward traveling field at the reference plane can be described as

$$
F^{-}\left(t, z_{n}\right)=\sqrt{R_{2}} F^{+}\left(t, z_{n}\right)
$$

where $R_{2}$ denotes the back facet reflection of the device cavity.

By solving (5) recursively on each reference plane in the time domain and using the boundary conditions (7) and (8), high-speed dynamic properties of various optical semiconductor devices can be described. Time-varying phenomena with durations down to the order of picoseconds are described by the model, provided that the slowly varying envelope approximation is fulfilled: 
To evaluate the carrier density $N_{m_{ \pm}}$in subsection $m_{+}$and $m_{-}$, the usual rate equation is employed [10]:

$$
\begin{aligned}
\frac{d N_{m_{ \pm}}}{d t}= & \frac{I_{m_{ \pm}}}{e d_{m_{ \pm}} w_{m_{ \pm}} L_{\mathrm{sec}}} \\
& -R_{m_{ \pm}}-g_{M, m_{ \pm}} V_{g, m_{ \pm}} S_{m_{ \pm}}
\end{aligned}
$$

Here, $g_{M, m_{ \pm}}$is the material gain, $I_{m_{ \pm}}$is the bias current, $w_{m_{ \pm}}$is the width, and $d_{m_{ \pm}}$is the thickness of the active layer in subsection $m_{+}$and $m_{-}$, respectively. $e$ denotes the elementary charge. For the spontaneous recombination rate $R_{m_{ \pm}}$, the following relation has been used [11]:

$$
R_{m_{ \pm}}=c_{1, m_{ \pm}} N_{m_{ \pm}}+c_{2, m_{ \pm}} N_{m_{ \pm}}^{2}-c_{3, m_{ \pm}} N_{m_{ \pm}}^{3}
$$

where $c_{1, m_{ \pm}}, c_{2, m_{ \pm}}$, and $c_{3, m_{ \pm}}$are recombination coefficients. The last term in (9) represents the stimulated recombination rate per unit volume, while $S_{m_{ \pm}}$denotes the total average spontaneous and signal photon density in subsection number $m_{+}$and $m_{-}$.

\section{THE DBR LASER AS A WAVELENGTH CONVERTER}

All-optical wavelength converters, which transform information from one wavelength to another, will be important components in future optical WDM communication networks, either to perform photonic space switching [1] or simply to increase the capacity and flexibility of the networks [13]. This section describes how a change in the signal wavelength can be obtained by using a DBR laser as the wavelength conversion element.

Wavelength conversion by the DBR laser is accomplished as follows. An optical signal carrying information in amplitudeshift keying (ASK) format with wavelength $\lambda_{\text {in }}$ is injected into the DBR laser that is biased above threshold and oscillating at wavelength $\lambda_{\text {out }}$. The injected input channel depletes the carriers of the gain section, and thereby controls the oscillation of the laser. The following two conversion schemes are possible.

1) The depletion of carriers will modulate the output power $P_{\text {out }}$ at $\lambda_{\text {out }}$, which therefore carries the same information as the input signal at $\lambda_{\text {in }}$. Thus, the information has been transferred from wavelength $\lambda_{\text {in }}$ to wavelength $\lambda_{\text {out }}$ while maintaining the ASK format. In the following, this conversion principle will be denoted ASK-to-ASK conversion.

2) The depletion of carriers also affects the round-trip phase change of the optical field in the cavity due to the change of the refractive index, which leads to modulation of the lasing frequency. Wavelength conversion can then be performed between an ASK signal format at wavelength $\lambda_{\text {in }}$ and an FSK (frequency-shift keying) format at wavelength $\lambda_{\text {out }}$. This technique will be denoted ASK-to-FSK conversion, and does, however, require optical demodulation from FSK to ASK before detection in a direct detection system.

The DBR wavelength converter is shown schematically in Fig. 2. Here, $R_{1}$ and $R_{2}$ denote the facet reflectivities, while $\kappa$ is the coupling coefficient of the grating section. $L_{g}, L_{p}$, and $L_{B}$ are the lengths of the gain section, the phase section, and the grating section, each with bias currents $I_{g}, I_{p}$, and $I_{B}$, respectively.

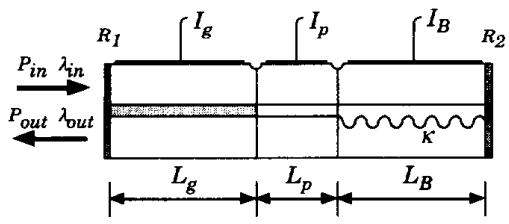

Fig. 2. Schematic of a DBR wavelength converter.

The output wavelength $\lambda_{\text {out }}$, determined by the Bragg condition, can be tuned either by alternating the phase section current $I_{p}$ or by changing the current to the grating section $I_{B}$. Two parameters are of importance for the wavelength tuning of the converter: the tuning range and the tuning speed. To cover as many channels as possible in a WDM network, the tuning range of the converter should be large. The tuning speed, i.e., how fast the output wavelength can be changed, is also important for some switching applications, e.g., fast packet switching [1].

\section{APPLicAtion OF THE MODEL}

In this section, we will consider the modeling of DBR converters in the case of ASK-to-ASK as well as ASK-toFSK wavelength conversion. The performance of a wavelength converter is here assessed in terms of the output extinction ratio, the frequency deviation, and the rise and fall time of the converted signal.

\section{A. Numerical Procedure}

All the equations required for a numerical analysis of the field distribution in the DBR laser are given in Section II and the Appendix. It is assumed that no spontaneous emission will be generated in the phase section and the Bragg section of the laser, and the amplified spontaneous emission (ASE) can then be implemented as suggested by [12].

The sequence of computing is shown in the flowchart in Fig. 3. Initially, a steady-state solution for the carrier density in each of the $n$ subsections of the model is found at the frequency for the oscillating mode. The mode having the highest round-trip gain as well as fulfilling the condition

$$
\phi=2 L_{\mathrm{sec}} \sum_{i=1}^{n} \beta_{i}=2 \pi p, \quad p \in\{1,2, \cdots\}
$$

oscillates. $\phi$ denotes the round-trip phase change of the optical field in the cavity. The phase condition (11) is accurate only if no grating section is included. For simplicity, however, (11) has been used in the following to find the lasing wavelength and calculate the chirp. Having found the mode for which (11) is fulfilled, the steady-state solution of the carrier density is found, and the dynamic analysis can begin.

The time $t$ is set to zero and a change of the operation conditions is performed. Then the rate equation (9) is applied to each subsection to find the new carrier density. For this carrier density, the forward and backward traveling fields and the photon density are found for the mode fulfilling (11). This procedure repeats itself in time steps of $\Delta t$ until the desired time is reached. 


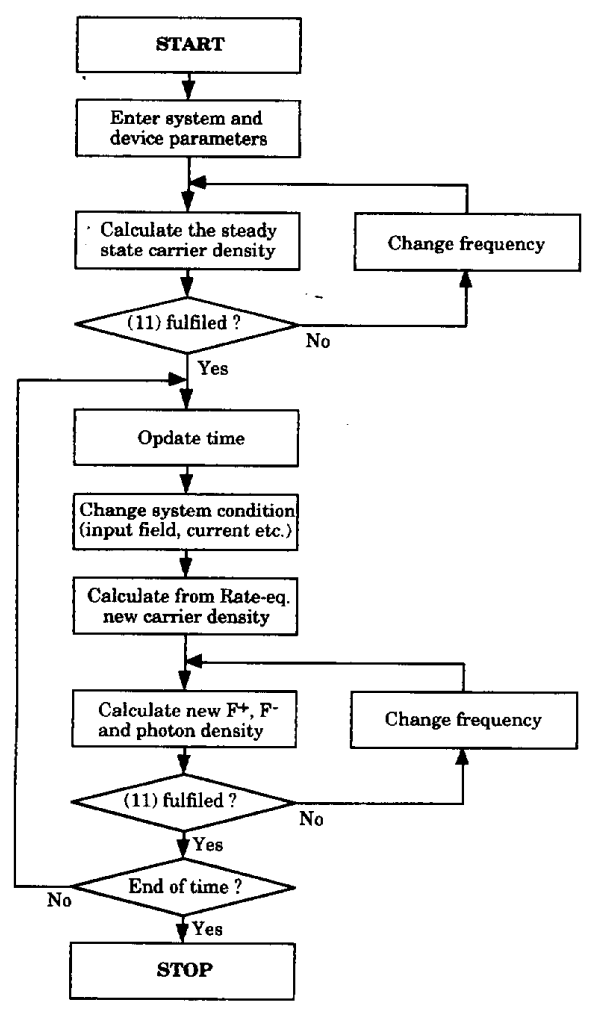

Fig. 3. Flowchart for calculating a dynamic response of the DBR converter.

\section{B. Numerical Results}

The parameters for the DBR converter under investigation are listed in Tables I and II. Here, we do not focus on the tuning characteristics of the DBR converter, so the phase section and the Bragg section currents are set to $I_{p}=I_{B}=0$. This leads to a Bragg wavelength of $1560 \mathrm{~nm}$ and an output wavelength $\lambda_{\text {out }}$ near the Bragg wavelength, for which (11) is fulfilled.

The back facet $R_{2}$ should be AR-coated to eliminate feedback from the mirror, and thereby reduce the influence of residual Fabry-Perot resonances on the injected signal [14]. Therefore, in all examples presented, $R_{2}$ is set to zero.

To demonstrate the dynamic behavior of the model, a dynamic response at conditions of large-signal current modulation is shown in Fig. 4. The gain section current is switched from just below 1 to 3.5 times the threshold, which is $28 \mathrm{~mA}$ for the considered device with cleaved input facet $\left(R_{1}=36 \%\right)$ and $\kappa L_{B}=1$. The transient response shows damped relaxation oscillations with a resonance frequency close to $5 \mathrm{GHz}$. The oscillations decay in about $1.5 \mathrm{~ns}$ to reach the steady state. Fig. 4 also shows that the output power is contained by a turn-on delay of approximately 150 ps.

The signal power distribution of the lasing mode along the longitudinal position of the DBR laser is shown in Fig. 5. The calculation is performed with the same device as above and a gain section current of 1.5 times the threshold current. The discontinuity between the gain section and phase section is caused by coupling loss due to the change of the waveguide dimension. In the Bragg section, it is obvious that the resulting
TABLE I

List of Parameter VAlues

\begin{tabular}{|c|c|c|c|c|c|}
\hline & Parameter & $\begin{array}{l}\text { Gain } \\
\text { Section }\end{array}$ & $\begin{array}{l}\text { Phase } \\
\text { Section }\end{array}$ & $\begin{array}{l}\text { Bragg } \\
\text { Section }\end{array}$ & \\
\hline$a$ & $\begin{array}{l}\text { Differential } \\
\text { gain }\end{array}$ & $3.00 \cdot 10^{-20}$ & 0 & 0 & $\left(m^{2}\right)$ \\
\hline$\gamma$ & Gain factor & $2.70 \cdot 10^{-24}$ & 0 & 0 & $\left(m^{-1} \cdot s^{2}\right)$ \\
\hline$\Gamma$ & $\begin{array}{l}\text { Confinement } \\
\text { factor }\end{array}$ & 0.31 & 0.54 & 0.53 & \\
\hline$\alpha_{i}$ & Internal loss & $3.00 \cdot 10^{3}$ & $3.00 \cdot 10^{3}$ & $4.00 \cdot 10^{3}$ & $\left(m^{-1}\right)$ \\
\hline$d$ & $\begin{array}{l}\text { Waveguide } \\
\text { thickness }\end{array}$ & 0.15 & 0.30 & 0.30 & $(\mu \mathrm{m})$ \\
\hline$w$ & $\begin{array}{l}\text { Waveguide } \\
\text { width }\end{array}$ & 2.00 & 2.00 & 2.00 & $(\mu \mathrm{m})$ \\
\hline$\alpha_{H}$ & $\begin{array}{l}\text { Linewidth } \\
\text { enhancement } \\
\text { factor }\end{array}$ & 5.00 & 3.00 & 3.00 & \\
\hline$n_{r}$ & $\begin{array}{l}\text { Reference } \\
\text { refractive index }\end{array}$ & 3.24 & 3.21 & 3.20 & \\
\hline$\Lambda$ & $\begin{array}{l}\text { Corrugation } \\
\text { period }\end{array}$ & 0 & 0 & $2.42 \cdot 10^{-7}$ & (m) \\
\hline$l$ & $\begin{array}{l}\text { Order of } \\
\text { grating }\end{array}$ & 0 & 0 & 1.00 & \\
\hline$\kappa$ & $\begin{array}{l}\text { Coupling } \\
\text { coefficient }\end{array}$ & 0 & 0 & $2.00 \cdot 10^{3}$ & $\left(m^{-1}\right)$ \\
\hline$\partial \kappa / \partial \omega$ & $\begin{array}{l}\text { Coupling shift } \\
\text { coefficient }\end{array}$ & 0 & 0 & $1.64 \cdot 10^{-15}$ & $\left(m^{-1} \cdot s\right)$ \\
\hline$\partial n / \partial \omega$ & $\begin{array}{l}\text { Refractive } \\
\text { index shift } \\
\text { coefficient }\end{array}$ & $1.00 \cdot 10^{-16}$ & $1.00 \cdot 10^{-16}$ & $1.00 \cdot 10^{-16}$ & (s) \\
\hline$\partial \omega / \partial N$ & $\begin{array}{l}\text { Frequency shift } \\
\text { coefficient }\end{array}$ & $2.12 \cdot 10^{-11}$ & $2.12 \cdot 10^{-11}$ & $2.12 \cdot 10^{-11}$ & $\left(\mathrm{~s}^{-1} \cdot \mathrm{m}^{3}\right)$ \\
\hline$c_{1}$ & $\begin{array}{l}\text { Recombination } \\
\text { coefficient }\end{array}$ & $1.50 \cdot 10^{8}$ & $1.68 \cdot 10^{8}$ & $1.68 \cdot 10^{8}$ & $\left(s^{-1}\right)$ \\
\hline$c_{2}$ & $\begin{array}{l}\text { Recombination } \\
\text { coefficient }\end{array}$ & $4.00 \cdot 10^{-16}$ & $0.28 \cdot 10^{-16}$ & $0.28 \cdot 10^{-16}$ & $\left(\mathrm{~m}^{3} \cdot \mathrm{s}^{-1}\right)$ \\
\hline$c_{3}$ & $\begin{array}{l}\text { Recombination } \\
\text { coefficient }\end{array}$ & $5.00 \cdot 10^{-41}$ & $5.24 \cdot 10^{-42}$ & $5.24 \cdot 10^{-42}$ & $\left(\mathrm{~m}^{6} \cdot \mathrm{s}^{-1}\right)$ \\
\hline
\end{tabular}

TABLE II

List of Parameter Values

\begin{tabular}{|c|c|c|c|}
\hline & Parameter & Value & \\
\hline$N_{0}$ & $\begin{array}{l}\text { Carrier density at } \\
\text { transparency }\end{array}$ & $0.90 \cdot 10^{24}$ & $\left(m^{-3}\right)$ \\
\hline$N_{r}$ & $\begin{array}{l}\text { Reference carrier } \\
\text { density }\end{array}$ & $3.00 \cdot 10^{24}$ & $\left(m^{-3}\right)$ \\
\hline$\omega_{r}$ & $\begin{array}{l}\text { Reference } \\
\text { frequency }\end{array}$ & $1.22 \cdot 10^{15}$ & $(\mathrm{rad} / \mathrm{s})$ \\
\hline$L_{g}$ & $\begin{array}{l}\text { Gain section } \\
\text { length }\end{array}$ & 300 & $(\mu \mathrm{m})$ \\
\hline$L_{p}$ & $\begin{array}{l}\text { Phase section } \\
\text { length }\end{array}$ & 100 & $(\mu \mathrm{m})$ \\
\hline$L_{B}$ & $\begin{array}{l}\text { Bragg section } \\
\text { length }\end{array}$ & 500 & $(\mu \mathrm{m})$ \\
\hline$n$ & $\begin{array}{l}\text { Number of } \\
\text { subsections }\end{array}$ & 90 & \\
\hline
\end{tabular}

periodic perturbation of the refractive index provides feedback by means of backward Bragg scattering, which couples the forward and backward propagating waves.

To investigate the gain saturation effect in the DBR converter, a calculation of the carrier density as a function of the longitudinal position in the gain section is shown in Fig. 6 , with the input power as a parameter. Without injected optical input power, the carrier density decreases due to increasing spontaneous recombinations at the outer ends of the gain section. By injecting a high input power at wavelength 

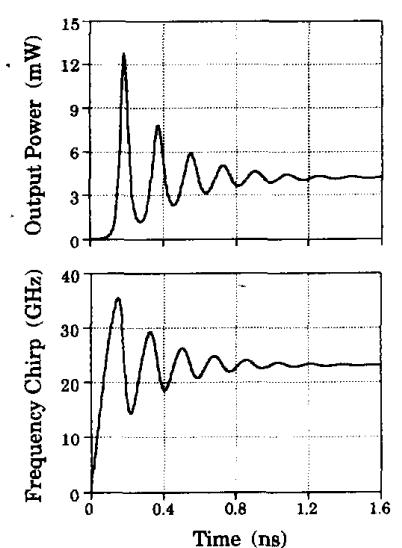

Fig. 4. Output power and frequency chirp versus time in case of a step function current modulation, switched from just below 1 to 3.5 times the threshold current.

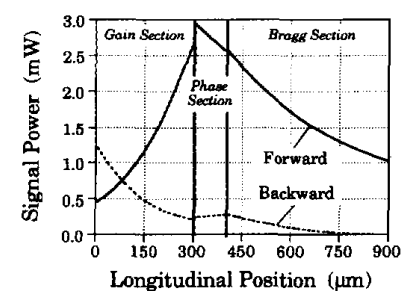

Fig. 5. Signal power distribution in the longitudinal position of the DBR converter. The driving source is the ASE generated in the gain section of the laser.

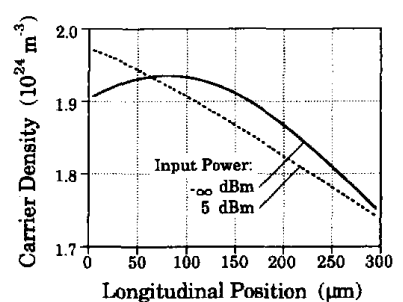

Fig. 6. Carrier density versus the longitudinal position of the gain section with injected input power as parameter. The wavelength of the injected $(\mathrm{CW})$ channel is $\lambda_{\text {in }}=1570 \mathrm{~nm}$ and the output wavelength is $\lambda_{\text {out }}=1560 \mathrm{~nm}$.

$\lambda_{\text {in }}=1570 \mathrm{~nm}$, stimulated recombinations caused by the input signal will be dominant, and the carrier density and thereby the modal amplitude gain decrease in the longitudinal direction of the gain section.

A good indication of the efficiency of the gain saturation can be obtained by calculating the output power level of the converted signal ( $\lambda_{\text {out }}=\sim 1560 \mathrm{~nm}$ ) versus input power at a wavelength $\lambda_{\text {in }}=1570 \mathrm{~nm}$ under static operation conditions. The result is seen in Fig. 7 with the front facet reflection $R_{1}$ as a parameter. For the cleaved input facet $\left(R_{1}=36 \%\right)$ and a gain section current of $I_{g}=50 \mathrm{~mA}$, an input power of $+5 \mathrm{dBm}$ is needed to reduce the output power by $3 \mathrm{~dB}$. By applying an AR coating so that $R_{1}=10 \%$, the needed input power is reduced to only $-1 \mathrm{dBm}$. This is due to a higher carrier concentration and thereby a higher gain, in addition to

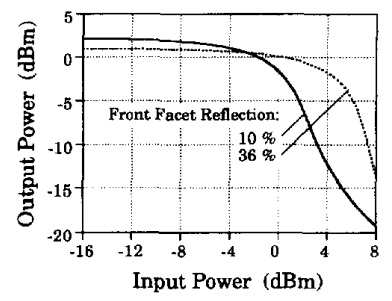

Fig. 7. Output power at wavelength $\lambda_{\text {out }}=1560 \mathrm{~nm}$ versus input power at wavelength $\lambda_{\text {in }}=1570 \mathrm{~nm}$ with the front facet reflection $R_{1}$ as parameter. Gain section current is $I_{g}=50 \mathrm{~mA}$.

a higher coupled input power into the cavity of the AR-coated DBR converter. It is also noted that the coated converter has superior output power in spite of a higher threshold current of $34 \mathrm{~mA}$. For an input channel with fixed input power and extinction ratio, Fig. 7 also indicates the obtainable output power and extinction ratio for the converted signal. As an example, in the case of $R_{1}=10 \%$, modulation of the output power between -12 and $2 \mathrm{dBm}$ is induced by injecting a signal with $3 \mathrm{dBm}$ peak power and a $10 \mathrm{~dB}$ extinction ratio. An improvement in extinction ratio of $4 \mathrm{~dB}$ is possible, which indicates the regenerative effect simultaneous to the wavelength conversion.

The remaining calculations are performed by injecting an optical step pulse, characterized by the average power $P_{\text {in }}$, the extinction ratio, and the wavelength $\lambda_{\text {in }}$. The wavelength conversion is simulated over approximately $10 \mathrm{~nm}$ from $\lambda_{\text {in }}=$ $1570 \mathrm{~nm}$ to $\lambda_{\text {out }}=\sim 1560 \mathrm{~nm}$.

In particular, the input power is of considerable importance since the injected optical signal should have a magnitude comparable to the power of the lasing mode to induce the desired gain saturation. Fig. 7 verifies that one way to reduce the needed input power is to apply an AR coating to the front mirror. Therefore, the following simulations are for a front facet reflection of $R_{1}=10 \%$. To illustrate the input power dependency, a calculation of the output extinction ratio in the case of ASK-to-ASK conversion, and the frequency deviation in the case of ASK-to-FSK conversion is shown in Fig. 8 versus average input power with the input extinction ratio as a parameter. An increasing output extinction ratio and frequency deviation are obtained for an input signal with increasing input power and extinction ratio. As an example, for an input extinction ratio of $10 \mathrm{~dB}$, an input power of at least $0 \mathrm{dBm}$ is required to avoid an extinction ratio degradation in the case of ASK-to-ASK conversion. For an input extinction ratio of $3 \mathrm{~dB}$, it is seen that the output extinction ratio exhibits a maximum for an input power of $3 \mathrm{dBm}$. This is because the input signal in the "low state" for higher power levels also contributes to the gain saturation. In the case of ASK-to-FSK conversion, the demodulation from FSK to ASK signal formats can be performed by, e.g., a Mach-Zehnder interferometer. This technique requires a frequency deviation at the output of the DBR converter at half the free spectral range of the Mach-Zehnder interferometer [2]. In practice, the demodulation requires a frequency modulation index of at least 1 (where a modulation index of 0.5 is the theoretical limit). Consequently, a conversion speed of $10 \mathrm{~Gb} / \mathrm{s}$ requires 


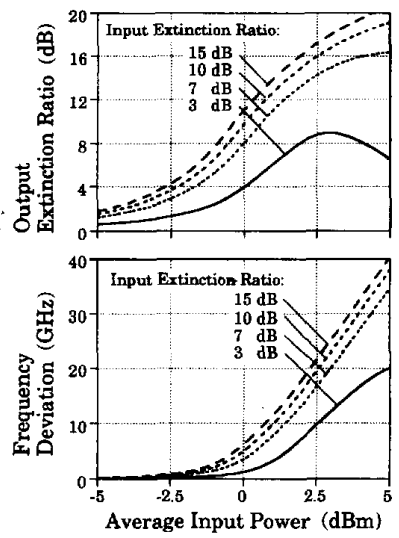

Fig. 8. Output extinction ratio and frequency deviation as a function of average input power with the input extinction ratio as parameter. The gain section current is $I_{g}=50 \mathrm{~mA}$, the input wavelength $\lambda_{\text {in }}=1570 \mathrm{~nm}$, and the output wavelength $\lambda_{\text {out }}$ is $1560 \mathrm{~nm}$.
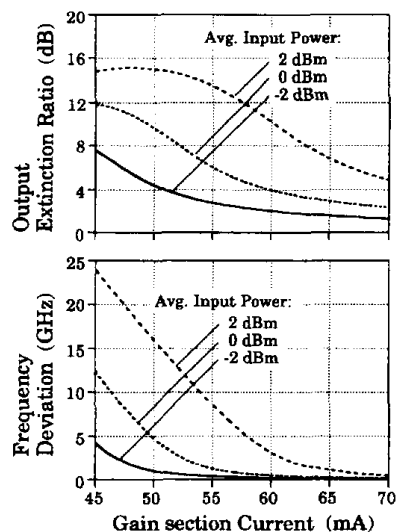

Fig. 9. Output extinction ratio and frequency deviation as function of gain section current $I_{g}$ with the average input power as parameter. The input extinction ratio is $10 \mathrm{~dB}$, the input wavelength $\lambda_{\text {in }}=1570 \mathrm{~nm}$, and the output wavelength $\lambda_{\text {out }}$ is $1560 \mathrm{~nm}$.

a frequency deviation of at least $10 \mathrm{GHz}$. According to Fig. 8 , this is obtained for an input signal of power approximately $1 \mathrm{dBm}$ and an extinction ratio of $10 \mathrm{~dB}$.

The input power needed to obtain efficient wavelength conversion can be reduced by decreasing the gain section current. However, the bias condition has a significant influence not only on the output extinction ratio and the frequency deviation, but also on the rise and fall times of the converted signal, which is illustrated in Figs. 9 and 10. Fig. 9 shows the output extinction ratio together with the frequency deviation as function of $I_{g}$ with the average input power as a parameter. A decreasing output extinction ratio with increasing current and decreasing input power is observed. For input powers of 0 and $+2 \mathrm{dBm}$, the calculation shows that the output extinction ratio exhibits a maximum value of 12 and $15 \mathrm{~dB}$, respectively, which occur when the laser is driven below threshold by the optical input signal. The calculation also shows that increasing frequency deviation is attained by decreasing the gain section current or increasing the input power.
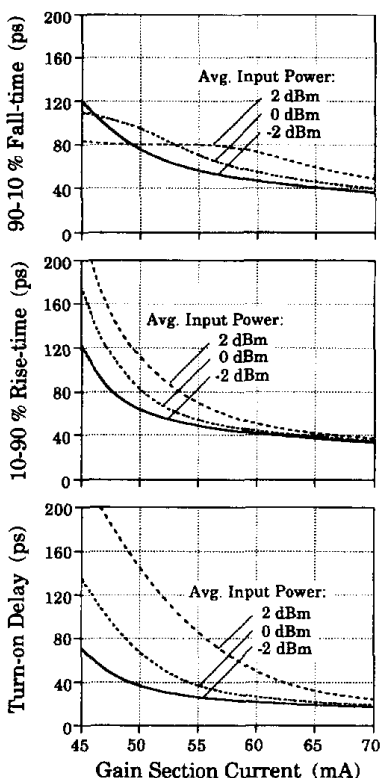

Fig. 10. Rise and fall times together with turn-on delay versus gain section current $I_{g}$. The average input power is parameter. The input extinction ratio is $10 \mathrm{~dB}$, the input wavelength $\lambda_{\text {in }}=1570 \mathrm{~nm}$, and the output wavelength $\lambda_{\text {out }}$ is $1560 \mathrm{~nm}$.

The operating speed of the DBR converter is determined by the relaxation frequency and the turn-on delay. A high relaxation frequency, which is caused by a high carrier density and a high photon density, results in short rise and fall times. This is confirmed by the results in Fig. 10, showing 10-90\% rise and fall times, together with turn-on delay of the converted signal as a function of the gain section current in the case of ASK-to-ASK conversion. Decreasing switching times are obtained for increasing bias current and input power. The fall time is fairly insensitive to variations in the bias current due to the high photon density from the injected input channel. The fall time is less than $100 \mathrm{ps}$ for $I_{g}>50 \mathrm{~mA}$. On the contrary, the rise time is more affected by the current level due to the lower photon density. For $I_{g}>60 \mathrm{~mA}$, the rise times are approximately $40 \mathrm{ps}$. However, strongly increasing rise times àre obtained for a decreasing gain section current due to the decreasing carrier density, and thereby a decreasing relaxation frequency. The turn-on delay, calculated as the $0-10 \%$ rise time, is also an important parameter in the discussion of the operating speed of the converter. This is seen from Fig. 10, showing that the turn-on delay, for an input power of $2 \mathrm{dBm}$, exceed the $10-90 \%$ rise time for a gain section current less than $55 \mathrm{~mA}$. This is because the laser is driven down below the threshold by the input field.

The regenerative effect of the extinction ratio is important for network applications, considering that the extinction ratio of the optical signal can degrade due to other components in the system. The extinction ratio improvement at the conversion is shown in Fig. 11 versus input extinction ratio with the average input power as a parameter. Improvements in extinction ratio of up to approximately $7 \mathrm{~dB}$ are obtainable for an input power of $2 \mathrm{dBm}$, and decrease for lower input 


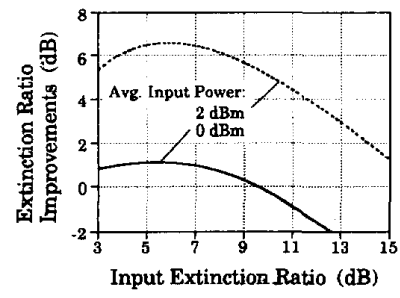

Fig. 11. Extinction ratio improvements as a function of the input extinction ratio with average input power as parameter. The wavelength conversion is performed from 1570 to $1560 \mathrm{~nm}$, and the gain section current is $I_{g}=50 \mathrm{~mA}$.

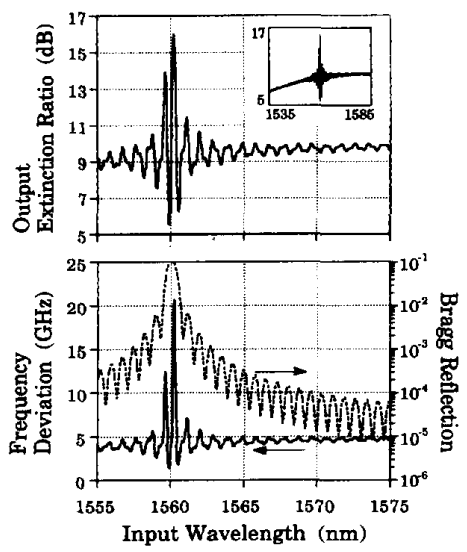

Fig. 12. Output extinction ratio and frequency deviation as a function of the wavelength of the injected channel. The Bragg reflection is also shown. The inset shows the extinction ratio over a wide wavelength span. The gain peak is at $1572 \mathrm{~nm}$ for the gain section current of $I_{g}=50 \mathrm{~mA}$.

powers. For an input power of $0 \mathrm{dBm}$, the improvement is only up to $1.2 \mathrm{~dB}$, and for extinction ratios larger than $9.5 \mathrm{~dB}$, a degradation in extinction ratio is expected. In both cases, the maximum improvement in extinction ratio is obtained for an input extinction ratio of $6 \mathrm{~dB}$.

Until now, the wavelength conversion has been performed over $10 \mathrm{~nm}$ from $\lambda_{\text {in }}=1570 \mathrm{~nm}$ to approximately $\lambda_{\text {out }}=$ $1560 \mathrm{~nm}$, but we have also investigated the influence of the injected input wavelength. Fig. 12 shows the output extinction ratio and frequency deviation, together with the reflection of the input signal from the Bragg section as a function of the input wavelength. The connection between Bragg reflection and conversion efficiency is clear: a peak in extinction ratio and frequency deviation is obtained near a peak in the Bragg reflection, together with the restriction that (11) is fulfilled for the injected field. Because of a relatively low $\kappa L_{B}$ of 1 , the Bragg reflection at the Bragg wavelength is only 0.12 , and by detuning the input wavelength relative to the Bragg wavelength, the fluctuations of the reflection are rather small. A higher value of $\kappa L_{B}$ results in larger fluctuations in the Bragg reflection and increases the wavelength sensitivity of the extinction ratio of the converted signal. Therefore, $\kappa L_{B}$ should be low to minimize the sensitivity of the input wavelength.

The possible range of the input wavelength is given by the gain bandwidth. This is verified by the inset in Fig. 12, also showing the extinction ratio, but over a wide input wavelength

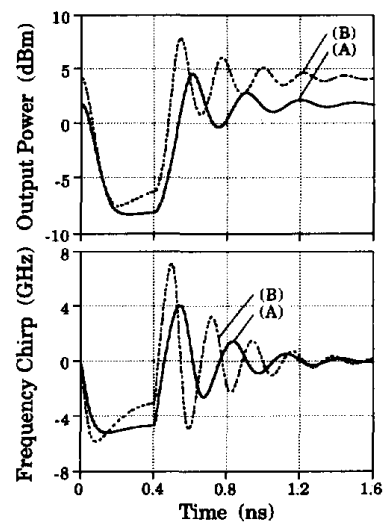

Fig. 13. Dynamic response of the DBR converter in case of (a) $P_{\mathrm{in}}=0$ $\mathrm{dBm}, I_{g}=50 \mathrm{~mA}$, and (b) $P_{\mathrm{in}}=2 \mathrm{dBm}, I_{g}=60 \mathrm{~mA}$. In both cases, a wavelength conversion from 1570 to $1560 \mathrm{~nm}$ is considered. The input channel turns to a "high state" at $t=0$ and returns to a "low state" at $t=0.4 \mathrm{~ns}$.

span. It is obvious that the extinction ratio follows the gain profile, which peaks at $1572 \mathrm{~nm}$ for the gain section current of $50 \mathrm{~mA}$. To provide the best conversion performance, the gain peak therefore should be close to the input wavelength.

Finally, an example of a dynamic response in the case of ASK-to-ASK and ASK-to-FSK conversion is shown in Fig. 13. At time $t=0$, an optical input step pulse is injected from the "low state" to the "high state" and turned off again to the "low state" after $0.4 \mathrm{~ns}$. First, it is noted from the calculation of the output power that the signal after the wavelength conversion is inverted in the case of the ASK-to-ASK scheme. This is not necessarily the case for the FSK detection scheme since it depends on the optical demodulation from the FSK-toASK signal format. Second, the calculation shows significantly faster rise and fall times in the case of ASK-to-FSK conversion because the frequency deviation follows the carrier density, contrary to the output power that follows the photon density. (a) is performed with an input average power of $0 \mathrm{dBm}$, an extinction ratio of $10 \mathrm{~dB}$, and a gain section current of $50 \mathrm{~mA}$. (b) is calculated for an input power of $2 \mathrm{dBm}$, an extinction ratio of $10 \mathrm{~dB}$, and a gain section current of $60 \mathrm{~mA}$. In both cases, the output extinction ratio is approximately $10 \mathrm{~dB}$ and the frequency deviation is $5 \mathrm{GHz}$. It is obvious that case (b), by virtue of the largest carrier and photon densities, results in the highest relaxation frequency, and thereby the shortest switching times.

\section{CONCLUSION}

A detailed numerical large-signal model based on timedependent coupled-wave equations is developed to calculate the static and transient behavior of DBR-type optical devices. The constitution of the model offers the possibility to simulate various kinds of semiconductor structures such as ordinary optical amplifiers, DFB and DBR lasers, or even more complex components. By dividing the cavity into several subsections, the nonuniform distribution of carrier and photon densities as well as that of the refractive index are found. 
Here, the model has been used to determine the influence of system and device parameters on the performance of DBR-wavelength converters. It has been shown that efficient wavelength conversion in the case of ASK-to-ASK as well as ASK-to-FSK signal formats can be obtained by carrier depletion in a DBR laser. From the modeling, the following can be concluded.

The front facet should AR-coated to reduce the required input power for inducing the sufficient carrier depletion. The back facet should also be AR-coated in order to reduce the influence of residual Fabry-Perot resonances on the injected signal. The value of $\kappa L_{B}$ should be low to minimize the fluctuations in the reflection from the Bragg region. This will decrease the wavelength dependency of the injected input signal. The gain peak at the operation current should be close to the wavelength of the injected input signal to maximize the carrier depletion effect, and thereby the output extinction ratio. The gain bandwidth should be large, to maximize the input wavelength range. The input wavelength $\lambda_{\text {in }}$ should not be too close to the Bragg wavelength in order to avoid a degradation in the extinction ratio of the signal. The input power $P_{\text {in }}$ should be the highest possible to induce the best performance of the wavelength conversion, and finally, a high input extinction ratio ensures a high output extinction ratio.

In conclusion, the model shows that efficient, ultrafast wavelength conversion with switching times less than 50 ps can be obtained as a result of the correct design and operation of the DBR converter.

\section{APPENDIX: \\ DERIVATION OF FIELD EQUATIONS (1)}

In the following, the mode coupled partial differential equations (1) for description of the field distribution in an optical semiconductor device will be derived. Only the fundamental tranverse mode is considered, and as shown in Fig. 1, the electric field $E\left(\omega, z_{m}\right)$ can be decomposed into right and left traveling fields $E\left(\omega, z_{m}\right)=E^{+}\left(\omega, z_{m}\right)+E^{-}\left(\omega, z_{m}\right)$. To find the field evolution in each direction, the one-dimensional wave equation in the frequency domain is used:

$$
\frac{\partial^{2} E^{ \pm}\left(\omega, z_{m}\right)}{\partial z^{2}}+K_{m_{ \pm}}^{2} E^{ \pm}\left(\omega, z_{m}\right)=0 .
$$

Here, $m_{-}$and $m_{+}$denote the subsections on the left and the right side on reference plane number $m$, respectively, and $K_{m_{ \pm}}$is the complex wavenumber in the subsections, which can be written as [15]

$$
K_{m_{ \pm}}^{2}=\beta_{m_{ \pm}}^{2}+j 2 \beta_{m_{ \pm}}^{2} g_{m_{ \pm}}^{2}+2 \beta_{m_{ \pm}} \kappa_{m_{ \pm}} e^{j \beta_{0, m_{ \pm}} z} .
$$

The parameters in (A2) will be explained in the following.

$\kappa_{m_{ \pm}}$is the grating coupling coefficient. In the case where subsection $m_{+}$or $m_{-}$does not contain a grating, $\kappa_{m_{+}}$or $\kappa_{m_{-}}$acquire the value zero.

$\beta_{0, m_{ \pm}}=l_{m_{ \pm}} \pi / \Lambda_{m_{ \pm}}$is the Bragg wavenumber, where $l_{m_{ \pm}}$ is the order of the grating and $\Lambda_{m_{ \pm}}$is the corrugation period in subsections $m_{+}$and $m_{-}$. Within each subsection, the grating is assumed homogeneous, i.e., $\kappa_{m_{ \pm}}$and $\Lambda_{m_{ \pm}}$are independent of $z$. $\beta_{m_{ \pm}}$denotes the real part of the wavenumber in subsection $m_{+}$and $m_{-}$and is given by

$$
\beta_{m_{ \pm}}=\frac{\omega}{c} n_{m_{ \pm}} .
$$

$c$ is the velocity of light in vacuum and $n_{m_{ \pm}}=n_{r}+\Delta n_{m_{ \pm}}$ is the effective refractive index in the subsections. $n_{r}$ is the refractive index at a reference carrier density $N_{r}$, and the linear part of the carrier-induced index change is related to the change in the carrier density by the following relation [9]:

$$
\Delta n_{m_{ \pm}}=-\frac{1}{2} \frac{c}{\omega_{r}} a_{m_{ \pm}} \Gamma_{m_{ \pm}} \alpha_{H, m_{ \pm}}\left(N_{m_{ \pm}}-N_{r}\right) .
$$

$\omega_{r}$ is a reference frequency, $N_{m_{ \pm}}$is the carrier density, $a_{m_{ \pm}}$is the differential gain factor, and $\Gamma_{m_{ \pm}}$is the confinement factor for the intensity in subsections $m_{+}$and $m_{-}$. The linewidth enhancement factor $\alpha_{H, m_{ \pm}}$is found experimentally to be a function of the carrier density as well as the wavelength [16]. For simplicity, $\alpha_{H, m_{ \pm}}$is assumed to be constant in this model. $g_{m_{ \pm}}$in (A2) is the modal amplitude gain in subsections $m_{+}$ and $m_{-}$, which can be written as

$$
g_{m_{ \pm}}=\frac{1}{2}\left(\Gamma_{m_{ \pm}} g_{M, m_{ \pm}}-\alpha_{L, m_{ \pm}}\right)
$$

where $\alpha_{L, m_{ \pm}}$is the internal loss per unit length caused by freeelectron absorption and scattering. The material gain $g_{M, m_{ \pm}}$ in (A5) is modulated as [17]

$$
g_{M, m_{ \pm}}=a_{m_{ \pm}}\left(N_{m_{ \pm}}-N_{0}\right)-\gamma_{m_{ \pm}}\left(\omega-\omega_{p, m_{ \pm}}\right)^{2} .
$$

$\gamma_{m_{ \pm}}$is a constant gain parameter, $N_{0}$ is the carrier density at transparency, and $\omega_{p, m_{ \pm}}$is the gain peak wavelength, which is assumed to shift linearly with the carrier density [11]

$$
\omega_{p, m_{ \pm}}=\omega_{r}+\frac{\partial \omega_{p}}{\partial N}\left(N_{m_{ \pm}}-N_{r}\right) \text {. }
$$

The reference frequency $\omega_{r}$ is the gain peak frequency at the reference carrier density $N_{r}$ and $\partial \omega_{p} / \partial N$ is assumed to be constant.

At reference plane number $m$, the left and right traveling fields take the form

$$
\begin{aligned}
E^{ \pm}\left(\omega, z_{m}\right)= & \psi^{ \pm}\left(\omega-\omega_{c}, z_{m}\right) e^{\mp j \beta_{m_{ \pm}} z_{m}} \\
& +\psi^{ \pm}\left(-\omega-\omega_{c}, z_{m}\right)^{*} e^{ \pm j \beta_{m_{ \pm}} z_{m}} .
\end{aligned}
$$

Here, $\omega_{c}$ is the carrier frequency and the (low-frequency) Fourier spectra $\psi^{ \pm}(\Omega, z)$ are given by

$$
\psi^{ \pm}(\Omega, z)=\int_{-\infty}^{\infty} F^{ \pm}(t, z) e^{-j \Omega t} d t
$$

$F^{ \pm}(t, z)$ are the slowly varying complex envelope functions in the time domain.

Inserting (A2) and (A8) in the one-dimensional wave equation (A1) and neglecting the second-order derivatives of $E^{ \pm}\left(\omega, z_{m}\right)$ with respect to $\omega$, we obtain

$$
\begin{aligned}
& \frac{\partial \psi^{ \pm}\left(\omega, z_{m}\right)}{\partial z} \\
& = \pm\left[g_{m_{ \pm}}+\left(\frac{\Gamma_{m_{ \pm}}}{2} \frac{\partial g}{\partial \omega}-j \frac{\partial \beta}{\partial \omega}\right)\left(\omega-\omega_{c}\right)\right] \psi^{ \pm}\left(\omega, z_{m}\right) \\
& \quad \mp j\left[\kappa_{m_{ \pm}}+\frac{\partial \kappa}{\partial \omega}\left(\omega-\omega_{c}\right)\right] \\
& \cdot e^{ \pm 2 j\left(\beta_{m_{ \pm}}-\beta_{0, m_{ \pm}}\right) z_{m}} \psi^{ \pm}\left(\omega, z_{m}\right)
\end{aligned}
$$


Using the relation (A9) between the Fourier spectra and the slowly varying envelopes, a set of partial differential equations in the time domain will be obtained:

$$
\begin{aligned}
& \frac{\partial F^{ \pm}\left(t, z_{m}\right)}{\partial z} \\
& \mp\left[g_{m_{ \pm}}-j\left(\frac{\Gamma_{\dot{m}_{ \pm}}}{2} \frac{\partial g}{\partial \omega}-j \frac{\partial \beta}{\partial \omega}\right) \frac{\partial}{\partial t}\right] F^{ \pm}\left(t, z_{m}\right) \\
& =\mp\left[\kappa_{m_{ \pm}}-\frac{\partial \kappa}{\partial \omega} \frac{\partial}{\partial t}\right] e^{ \pm 2 j\left(\beta_{m_{ \pm}}-\beta_{0, m_{ \pm}}\right) z_{m}} F^{\mp}\left(t, z_{m}\right) .
\end{aligned}
$$

This set, which is similar to (1), is the theoretical framework for the dynamic description of the electrical field in the optical semiconductor device.

\section{REFERENCES}

1] J. M. Gabriagues et al. "Exploitation of the wavelength domain for photonic switching in the IBCN," in Proc. ECOC/IOOC'91, Paris, France, Sept. 1991, invited paper Tu.C3.1, pp. 59-66.

[2] B. Mikkelsen et al., "Demonstration of all-optical wavelength conversion of high-speed signals in coherent- and direct-detection systems," in Proc. OFC/IOOC'93, Santa Barbara, CA, Feb. 1993, paper TuH5, pp. 34-35.

[3] G. Grosskopf et al., "140 Mbit/s DPSK transmission using an all-optical frequency converter with a $4000 \mathrm{GHz}$ converter range," Electron. Lett., vol. 24, pp. 1106-1107, 1988.

[4] T. Durhuus et al., "High speed all-optical gating using two section semiconductor optical amplifier structure," in Proc, CLEO'92, Anaheim, CA, May 1992, paper CThS4, pp. 552-553.

[5] T. Durhuus et al., " $2.5 \mathrm{~Gb} / \mathrm{s}$ optical gating with high on/off ratio by use of SOAs in Mach-Zehnder-interferometers," in Proc. CLEO'93, Baltimore, MD, May 1993, paper CThH3, p. 414.

[6] K. Kondo et al., "Efficient optical inverter operation of a wavelength conversion laser," in Proc. IOOC'89, Kobe, Japan, July 1989, paper 19C4-1, pp. 130-131.

[7] $O$. Hildebrand et al., "The integrated interferometric injection laser $(Y$ laser): One device concept for various system applications," in Proc. ECOC/IOOC'91, Paris, France, Sept. 1991, invited paper Tu.A5, pp. $39-46$.

[8] A. J. Lowery, A. Keating, and C. N. Murtonen, "Modeling the static and dynamic behavior of quarter-wave shifted DFB lasers," IEEE J. Quantum Electron., vol. 28, pp. 1874-1883, Sept. 1992.

[9] L. M. Zhang and J. E. Carrol, "Large-signal dynamic model of the DBR laser," IEEE J. Quantum Electron., vol. 28, pp. 604-611, Mar. 1992.

[10] G. P. Agrawal and N. K. Dutta, Long-Wavelength Semiconductor Lasers. New York: Van Nostrand Reinhold, 1986.

[11] T. Durhuus et al., "Detailed dynamic model for semiconductor optical amplifiers and their crosstalk and intermodulation distortion," $J$. Lightwave Technol., vol. 10, pp. 1056-1065, Aug. 1992.

[12] M. J. Adams et al., "Analysis of semiconductor laser optical amplifiers," Proc. IEE, part J, vol. 132, pp. 58-63, Feb. 1985.

[13] A. A. M. Saleh, "Wavelength-division multiplexing," in Proc. OFC/IOOC'92, San Jose, CA, Feb. 1992, paper ThC1, p. 99

[14] C. Braagaard et al. "Optimisation of DBR wavelength converters," in Proc. ECOC/IOOC'93, Montreux, France, Sept. 1993, paper Tu 18, pp. 237-240.

[15] H. Kogelnik and C. V. Shank, "Coupled-wave theory of distributed feedback lasers," J. Appl. Phys., vol. 43, pp. 2327-2335, May 1972.

[16] N. Storkfelt et al., "Measurement of carrier lifetime and linewidth enhancement factor for $1.5-\mu \mathrm{m}$ ridge-waveguide laser amplifier," IEEE Photon. Technol. Lett., vol. 3, pp. 632-634, July 1991.

[17] I. D. Henning et al. "Performance predictions from a new optical amplifier model," IEEE J. Quantum Electron., vol. QE-21, pp. 609-613, June 1985.

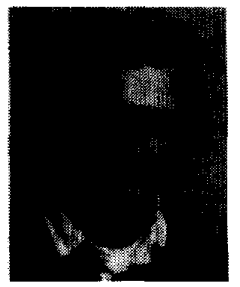

Carsten Braagaard was born in Odense, Denmark, in 1966 . He received the M.Sc. degree from the Technical University of Denmark (TUD), Copenhagen, in 1992.

$\mathrm{He}$ is currently working as a Research Associate at the Electromagnetics Institsute at TUD. His field of interest is the modeling of lasers and optical amplifiers as functional devices for photonic systems.

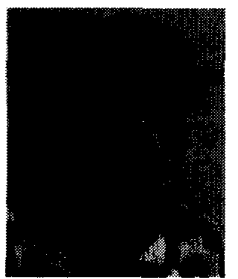

Benny Mikkelsen was born in Sdr. Nissum, Denmark, in 1960 . He received the M.Sc. degree from the Technical University of Denmark (TUD), Copenhagen, in 1987.

He is currently working toward the Ph.D. degree at the Electromagnetics Institute at TUD. His field of interest is optical amplifiers and their applications in optical networks.

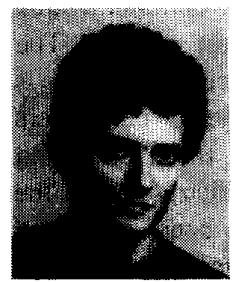

Terji Durhuus was born in Tórshavn, Faroe Islands, in 1962. He received the M.Sc. degree from the Technical University of Denmark (TUD), Copenhagen, in 1989.

He is currently working toward the Ph.D. degree at the Electromagnetics Institute. His field of interes is optical amplifiers, with emphasis on nonlinearities in semiconductor optical amplifiers.

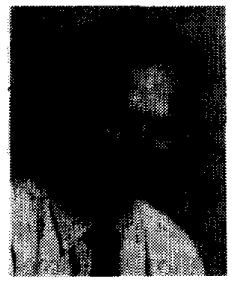

Kristian E. Stubkjaer (''80-M'81) was born in Denmark in 1953. He received the M.Sc. and Ph.D. degrees from Technical University of Denmark (TUD), Copenhagen, in 1977 and 1981 , respectively. From 1979 to 1981 he studied at the Tokyo Institute of Technology, Tokyo, Japan, with a scholarship from the Japanese Government.

From 1981 to 1982 he was in the military service at the Danish Defence Research Establishment, Copenhagen. From 1982 to 1983 he was a Visiting Scientist at the IBM T. J. Watson Research Center, Yorktown Heights, NY. He is now an Associate Professor at the Electromagnetics Institute, TUD, where he is working in the field of optical communication. From 1985 to 1990 he was Director of the Electromagnetics Institute, and currently he is serving as Chairman of the Electromechanica Committee under the Technical Research Council (Danish Ministry for Education). 